schen Handelsliberalisierung ("Von Miami nach Belo Horizonte: Stolpersteine auf dem Weg zu einer panamerikanischen Freihandelszone"). Stolpersteinen auf einem weit beschwerlicheren Weg widmet sich Margret Johannsen ("Der nahöstliche Friedensprozeß in der Sackgasse"), ein - leider immer noch nicht wesentlich verändertes - düsteres Bild zeichnend von dem, was vor wenigen Jahren noch so optimistisch mit dem Nobelpreissharing für Yassir Arafat, Shimon Peres und den unvergessenen Yitzhak Rabin honoriert worden war. Mit dem hochinteressanten Beitrag von Robert Kappel, der einer stärkeren politischen Einbettung Südafrikas in das regionale Umfeld das Wort redet ("Regionalmacht Südafrika: Das schwierige Erbe der Apartheid"), schließt der redaktionelle Teil des Bandes. Es folgen als Anhang neben der eingangs erwähnten Chronik (1. Juli 1996 - 30. Juni 1997) noch das verdienstvoll thematisch bzw. nach Ländern gegliederte Gesamtregister aller seit 1983 erschienen Beiträge und last not least die Autorenangaben.

Wie schon früher gelegentlich angedeutet: Das Jahrbuch Dritte Welt ist grundsätzlich nichts zum schnurstracks von-vorn-bis-hinten-Durchlesen. Es eignet sich vielmehr und weit besser, vor allem seiner den Beiträgen angefügten Literaturempfehlungen wegen, als profunde Einstiegs-Quelle. Ihr bleibt auch künftig weiteres Sprudeln zu wünschen.

Karl-Andreas Hernekamp

\title{
Ferdinand Fromholzer
}

\section{Consideration}

US-amerikanisches Recht im Vergleich zum deutschen

Studien zum ausländischen und internationalen Privatrecht, Band 57

Mohr Siebeck Verlag, Tübingen, 1997, 381 S., DM 138,--

Die consideration ist eines der besonderen Rechtsinstitute des common law. Für Juristen, die mit den Traditionen des common law nicht vertraut sind, ist es oft schwer, ihren Inhalt, Hintergrund und Anwendungsbereich zu erfassen. Ursache hierfür ist vor allem das Fehlen einer vergleichbaren Rechtsfigur in den kontinentaleuropäischen Rechtsordnungen. Ihrem Kern nach stellt die aus dem englischen common law stammende consideration-Lehre für die Verbindlichkeit vertraglicher Absprachen das Erfordernis auf, daß jedem Versprechen, eine Leistung zu erbringen, eine Gegenleistung gegenüberstehen muß.

Dieses Prinzip führt in einer Vielzahl von Fallgruppen zu Problemen, die Fromholzer in seiner von Professor Dr. Reinhard Zimmermann in Regensburg betreuten Dissertation ausführlich untersucht und mit entsprechenden deutschen Konstellationen vergleicht. Beispielhaft seien hier das unentgeltliche Versprechen, der Schulderlaß oder die nachträgliche Vertragsänderung genannt. Das Ziel der Arbeit geht jedoch über diesen Vergleich hinaus: Der Autor stellt einleitend die These auf, daß sich die Rechtssysteme immer stärker annä- 
hern (S. 1-7). Die Konvergenz von common law und kontinentaleuropäischem civil law folge daraus, daß die Ergebnisse auch dann übereinstimmten, wenn die consideration im anglo-amerikanischen Recht im Mittelpunkt stehe und man daher im Hinblick auf die Eigenart dieser Rechtsfigur Abweichungen erwarten könnte. Dieser Ausgangspunkt ist sicherlich interessant, wirft aber zugleich die Frage auf, inwiefern sich die Annäherung zweier Rechtskreise tatsächlich anhand einer Rechtsfrage und ihrer Behandlung in zwei als repräsentativ angesehenen Rechtsordnungen nachweisen läßt. Die fast vollständige Vernachlässigung des englischen Rechts, in welchem das consideration-Prinzip seine Wurzeln hat, ist auch unter Hinweis auf die weitgehende Übereinstimmung mit der Rechtslage in den Vereinigten Staaten (S. 7) nur teilweise überzeugend, da sich gerade in diesem Bereich einige Abweichungen ergeben - man beachte nur die unterschiedliche Einordnung des promissory estoppel oder die neuere Rechtsprechung zur nachträglichen Vertragsänderung (vgl. K. Zweigert / H. Kötz, Einführung in die Rechtsvergleichung, S. 388-390) - Unterschiede, die die These von der Annäherung der Rechtskreise zumindest nicht zu untermauern vermögen.

Im Kernstück der Untersuchung werden die einzelnen Fallgruppen der consideration behandelt. Fromholzer hat hier eine Fülle von Material zusammengetragen und sich dabei auch ausführlich dem case law gewidmet. Bedauerlich ist in diesem Zusammenhang allerdings das Fehlen eines Registers zumindest des anglo-amerikanischen Fallmaterials; auch hätte die Darstellung zuweilen etwas gestrafft werden können, da sich manches aus der Länderzusammenfassung in der jedes Kapitel abschließenden "Vergleichenden Zusammenfassung" wiederholt. Inhaltlich können hier nur einige Aspekte beispielhaft gewürdigt werden. So wird einleitend die Frage behandelt, die sich auch dem kontinentaleuropäischen Juristen zuerst stellen wird: Ob nämlich die consideration ein Mechanismus ist, der ein angemessenes Verhältnis von Leistung und Gegenleistung gewährleisten soll. Fromholzer weist darauf hin, daß die Funktion der consideration gerade nicht in einer solchen Äquivalenzkontrolle liegt, da auch eine geringfügige Gegenleistung ausreicht (peppercorn theory, S. 21-29). Zugleich führt er aus, daß ein extremes Mißverhältnis von Leistung und Gegenleistung aus anderen Gründen - vor allem bei der Beeinträchtigung der Willensfreiheit - zu einer Unwirksamkeit des Vertrages führen kann (S. 27 f.). Dieser Gedanke, daß die consideration in einem engen Zusammenhang zu der Vermeidung unzulässiger Einflußnahme auf eine Vertragspartei und vergleichbarer Wertungen zu sehen ist, setzt sich sodann im Laufe der Abhandlung fort, etwa bei der Entlohnung für bereits bestehende Pflichten (Kapitel IV) oder bei Versprechen, die erst nach Vornahme einer entsprechenden Handlung abgegeben werden (past consideration, Kapitel V).

Dieser Zusammenhang mag für den mit dem common law nicht vertrauten Leser auf den ersten Blick nicht einleuchten, da sich der Einwand aufdrängt, weswegen das consideration-Prinzip dadurch eingeschränkt sein soll, daß eine weitere Wirksamkeitsvoraussetzung eingreift. Das Zusammenspiel von consideration und Billigkeitsgesichtspunkten läßt sich wiederum aus der englischen Tradition heraus erklären, wonach sich eine Partei wegen eines als unbillig empfundenen Verhaltens unter Umständen auf ihr eigentlich zustehende 
Rechtspositionen nicht berufen darf (equity). Hieraus resultieren auch die unscharfen Konturen der consideration, die notwendigerweise dazu führen, daß im deutschen Recht eine Vielzahl von Rechtsfragen gestreift wird, bei denen man eine Zusammenhang mit der consideration nicht vermutet hätte. Hierin liegt eine der Stärken dieses Werkes, da es dem Autor gelungen ist, eine Fülle von Beispielen für die Anwendung der consideration zusammenzutragen und vergleichbare Fälle aus Deutschland zu finden. Es soll aber auch nicht verschwiegen werden, daß angesichts der zahlreichen Details die ursprüngliche Funktion der consideration als Indiz für die Seriosität eines Versprechens zu sehr in den Hintergrund gerät. Dies stützt zwar die Aussage des Autors, daß es sich nicht um ein einheitliches Prinzip handelt (S. 356); eine ausführlichere Erläuterung der Grundstrukturen der consideration wäre dennoch hilfreich gewesen.

Seine Ausgangsthese sieht Fromholzer dadurch bestätigt, daß die deutschen Gerichte zu ähnlichen Ergebnissen gelangen, sich dabei zunehmend von der Einzelfallgerechtigkeit leiten lassen und daher auch methodisch der Rechtsprechung der Vereinigten Staaten annähern. Die wachsende Bedeutung von Fall- und Richterrecht könne somit dazu führen, daß sich die Rechtsordnungen zukünftig stärker untereinander beeinflussen, ein Ergebnis, welches von Fromholzer nachdrücklich befürwortet wird (S. 356). Es sei hier abschließend noch ein Einwand gegen dieses detailreiche und in vielfacher Hinsicht anregende Werk gestattet: Kann tatsächlich von einer Konvergenz gesprochen werden, wenn die Rechtsordnungen mehr oder weniger zufällig zu übereinstimmenden Ergebnissen gelangen, ohne daß dies unter einer bewußten Beachtung der jeweils fremden Rechtsordnung und ihrer Wertungen und Gedankengänge geschieht?

Maria Kasche

\section{Iris Breutz}

\section{Der Protest im Völkerrecht}

Hamburger Studien zum europäischen und internationalen Recht, Bd. 11

Duncker \& Humblot Verlag, Berlin, 1997, 202 S., DM 98,--

Die Autorin nimmt die verdienstvolle Aufgabe auf sich, den völkerrechtlichen Protest, ein in der neueren Völkerrechtsliteratur wenig behandeltes Phänomen, einer umfassenden Untersuchung zu unterziehen. Dabei unterteilt sie ihre Arbeit zunächst in drei Hauptkapitel, deren erstes die "Rechtliche Beurteilung des erhobenen Protestes" zum Thema hat, während das zweite, deutlich kürzere, sich mit den "Rechtliche[n] Probleme[n] des unterlassenen Protestes" beschäftigt. Das dritte Kapitel gibt (ausdrücklich ohne Anspruch auf Vollständigkeit) einen knappen Überblick über von der Bundesrepublik Deutschland seit 1949 abgegebene Proteste. Ein abschließendes "4. Kapitel" enthält die Zusammenfassung und Schlußbetrachtung. 